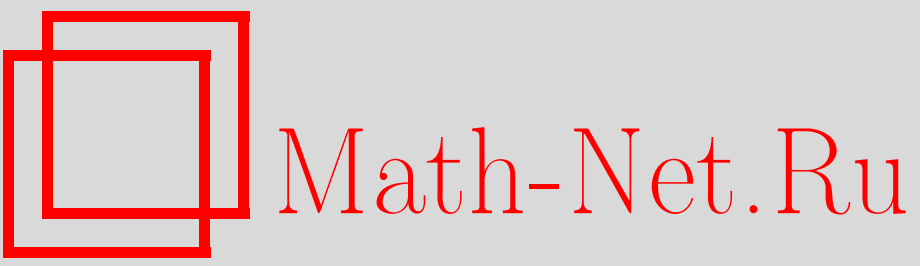

С. М. Сергеев, Схема квантования для модулярных q-разностных уравнений, TMФ, 2005, том 142, номер 3, 500-509

DOI: https://doi.org/10.4213/tmf1794

Использование Общероссийского математического портала Math-Net.Ru подразумевает, что вы прочитали и согласны с пользовательским соглашением

http://www . mathnet.ru/rus/agreement

Параметры загрузки:

IP : 54.205 .225 .156

26 апреля 2023 г., 03:26:23 
ТЕОРЕТИЧЕСКАЯ

И МАТЕМАТИЧЕСКАЯ

ФИЗИКА

Том 142, № 3

март, 2005

(C) 2005 г.

С. М. Сергеев*

\section{СХЕМА КВАНТОВАНИЯ ДЛЯ МОДУЛЯРНЫХ q-РАЗНОСТНЫХ УРАВНЕНИЙ}

Рассматриваются модулярные пары некоторых $q$-разностных уравнений второго порядка. Пример такой пары: $t-Q$ уравнения Бакстера для квантовой релятивистской цепочки Тоды в режиме сильной связи. Другой квантовомеханический пример: $q$-деф̆ормация уравнения Шредингера с гиперболическим потенциалом. Показано, что требование аналитичности волновой функции или функции Бакстера приводит к определенному набору трансцендентных уравнений на коэффициенты потенциала или трансфер-матрицы, решением которых является их дискретный спектр.

Ключевые слова: уравнения Бакстера, модулярная дуализация, режим сильной связи.

\section{1. ВВЕДЕНИЕ}

За последние несколько лет в теории квантовых вполне интегрируемых систем появилось несколько моделей, в которых алгеброй наблюдаемых является локальная алгебра Вейля со специальным значением вейлевского параметра $q[1],[2]$ :

$$
q=e^{2 \pi i b^{2}}, \quad b=e^{i \theta}, \quad 0<\theta<\frac{\pi}{2} .
$$

Ключевое свойство этого режима для $q$, называемого "режимом сильной связи", состоит в том, что комплексное сопряжение $q$ эквивалентно модулярному преобразованию Якоби, $q^{*}=e^{-2 \pi i b^{-2}}$ (в этой работе мы будем использовать звездочку как знак комплексного сопряжения).

Пусть операторы координаты х и импульса $\mathbf{p}$ алгебры Гейзенберга,

$$
\mathbf{x p}-\mathbf{p x}=\frac{i}{2 \pi}
$$

использованы для построения пары Вейля $\mathbf{u}, \mathbf{v}$,

$$
\mathbf{u}=e^{2 \pi b \mathbf{x}}, \quad \mathbf{v}=e^{2 \pi b \mathbf{p}}, \quad \mathbf{u} \mathbf{v}=q \mathbf{v} \mathbf{u},
$$

*Department of Theoretical Physics, Research School of Physical Sciences and Engineering, Australian National University, Canberra, Australia. E-mail: sergey.sergeev@anu.edu.au 
и модулярно дуальной (т.е. сопряженной) пары

$$
\mathbf{u}^{\dagger}=e^{2 \pi b^{-1} \mathbf{x}}, \quad \mathbf{v}^{\dagger}=e^{2 \pi b^{-1} \mathbf{p}}, \quad \mathbf{v}^{\dagger} \mathbf{u}^{\dagger}=q^{*} \mathbf{u}^{\dagger} \mathbf{v}^{\dagger} .
$$

Любой элемент первой пары, очевидно, коммутирует с любым элементом второй пары. Физическая интерпретация режима (1) состоит в том, что любой полином $\mathbf{J}(\mathbf{u}, \mathbf{v})$ коммутирует с себе сопряженным,

$$
\mathbf{J}(\mathbf{u}, \mathbf{v}) \mathbf{J}(\mathbf{u}, \mathbf{v})^{\dagger}=\mathbf{J}(\mathbf{u}, \mathbf{v})^{\dagger} \mathbf{J}(\mathbf{u}, \mathbf{v})
$$

т.е. можно говорить о коммутирующих эрмитовых операторах $\mathbf{J}+\mathbf{J}^{\dagger}$ и $i\left(\mathbf{J}-\mathbf{J}^{\dagger}\right)$, связанных с физическими гамильтонианами. Спектральной задачей является следуюшая пара уравнений на вектор $\langle\Psi|$ :

$$
\langle\Psi| \cdot \mathbf{J}(\mathbf{u}, \mathbf{v})=\langle\Psi| \cdot \mathbf{J}(\mathbf{u}, \mathbf{v})^{\dagger}=0 .
$$

Эти уравнения иногда называются уравнениями на квантовую кривую (поскольку в пределе $q \rightarrow 1$ уравнение $J(u, v)=0$ определяет классическую алгебраическую кривую). Уравнения (6) являются также базис-инвариантной записью $t$ - $Q$ уравнений Бакстера, $\langle\Psi| \equiv\langle Q|$. В рамках квантовой механики полиномы $\mathbf{J}$ и $\mathbf{J}^{\dagger}$ можно понимать как деформации оператора Шредингера $\mathbf{H}-E$.

Проблема разностных уравнений (6) состоит в том, что требование правильной асимптотики $\Psi$ в, например, координатном представлении (аналог квантовомеханического условия $\left.\Psi(x) \in L_{2}(\mathbb{R})\right)$, не фиксирует $\Psi$ однозначно и не приводит к квантованию спектpa.

В этой работе мы покажем, что если требование правильной асимптотики дополнить требованием аналитичности волновой функции в определенной полосе, то это приведет к однозначности волновой функции и к квантованию спектра. Уравнениями квантования спектра окажется система замысловатых уравнений в терминах трансцендентных, но хорошо определенных функций от коэффишиентов полиномов $\mathbf{J}$ и $\mathbf{J}^{\dagger}$. Благодаря трансцендентности, эта конечная система имеет бесконечное число решений.

Наше изложение мы начнем с квантовомеханического подхода, в рамках которого условие аналитичности является естественным, шаг за шагом построим волновую функцию и условия квантования спектра. Уравнение Бакстера для модулярной цепочки Тоды мы обсудим в заключение в предпоследнем разделе.

\section{2. КВАНТОВАЯ МЕХАНИКА q-ГИПЕРЭЛЛИПТИЧЕСКОГО МАЯТНИКА}

Мы будем рассматривать операторнозначньй полином $\mathbf{J}(\mathbf{u}, \mathbf{v})$ специального вида:

$$
\mathbf{J}(\mathbf{u}, \mathbf{v})=\mathbf{v}+\mathbf{v}^{-1}+T(\mathbf{u}),
$$

где “потенциал” (или, если угодно, трансфер-матрица) имеет вид

$$
T(u)=\lambda u^{-L} \sum_{j=0}^{2 L} t_{j} \cdot(-u)^{j}, \quad t_{0}=t_{2 L}=1,
$$


все $t_{j}$ и $\lambda$ - комплексные коэффициенты. Этот полином $\mathbf{J}$ может быть интерпретирован как $q$-деформация оператора Шредингера $\mathbf{j}=\mathbf{p}^{2}+V(\mathbf{x})-E$ с гиперболическим потенциалом. Заметим, что в пределе $q \rightarrow 1$ уравнение $J(u, v)=0$ определяет гиперэллиптическую кривую рода $g=2 L-1 \mathrm{c} g$ модулями $t_{j}$, и с точки зрения механики динамика $u$ и $v$ со скобкой $\{u, v\}=u v$ есть гиперэллиптический маятник.

Мы будем использовать координатное представление,

$$
\langle\Psi \mid x\rangle=\Psi(x), \quad\langle\Psi|\mathbf{x}| x\rangle=x \Psi(x), \quad\langle\Psi|\mathbf{p}| x\rangle=\frac{i}{2 \pi} \Psi^{\prime}(x)
$$

Из (3) и (4) следует

$$
\begin{array}{ll}
\langle\Psi|\mathbf{u}| x\rangle=e^{2 \pi x b} \Psi(x), & \left\langle\Psi\left|\mathbf{u}^{\dagger}\right| x\right\rangle=e^{2 \pi x b^{-1}} \Psi(x), \\
\langle\Psi|\mathbf{v}| x\rangle=\Psi(x+i b), & \left\langle\Psi\left|\mathbf{v}^{\dagger}\right| x\right\rangle=\Psi\left(x+i b^{-1}\right)
\end{array}
$$

Для краткости последующих формул для каждого $x \in \mathbb{C}$ положим

$$
u \equiv e^{2 \pi x b}, \quad \tilde{u} \equiv e^{2 \pi x b^{-1}} .
$$

Заметим, если $x$ комплексно, то $\tilde{u} \neq u^{*}$.

Обратимся теперь к уравнениям (6) в координатном представлении. Для полинома (7) они приобретают вид

$$
\begin{aligned}
\Psi(x+i b)+\Psi(x-i b)+\Psi(x) T(u) & =0, \\
\Psi\left(x+i b^{-1}\right)+\Psi\left(x-i b^{-1}\right)+\Psi(x) T^{*}(\tilde{u}) & =0,
\end{aligned}
$$

где $T^{*}$ означает комплексное сопряжение всех коэффициентов полинома Лорана $T$. $\Phi$ ункция $\Psi(x)$ при $x \in \mathbb{R}$ есть волновая функция, так что первое условие на $\Psi$ очевидно: $\Psi(x) \in L_{2}(\mathbb{R})$. Ясно, что сдвиг аргумента $\Psi$ в комплексную область, появляющийся во второй строке представления (10), должен пониматься как аналитическое продолжение физической функции $\left.\Psi(x)\right|_{x \in \mathbb{R}}$. В рамках квантовой механики реализация комплексных сдвигов - это разложение в ряд (напомним, $i b=i \cos \theta-\sin \theta)$ :

$$
\Psi(x+i b)=\sum_{n=0}^{\infty} \Psi^{(n)}(x-\sin \theta) \frac{(i \cos \theta)^{n}}{n !}
$$

которое хорошо определено тогда и только тогда, когда $\Psi(x)$ аналитична в полосе

$$
-\cos \theta<\operatorname{Im} x<\cos \theta
$$




\section{3. ГОЛОМОРФНЫЕ ЧАСТИ}

Путем небольшой модифицикации каждое из уравнений (12) может быть приведено к уравнению, имеющему голоморфное по $u^{ \pm 1}$ или по $\tilde{u}^{ \pm 1}$ решение. Введем обозначения

$$
t_{+}(u)=\frac{u^{L}}{\lambda} T(u)=\sum_{j=0}^{2 L} t_{j} \cdot(-u)^{j}, \quad t_{-}(u)=\frac{1}{u^{L} \lambda} T(u)=\sum_{j=0}^{2 L} t_{2 L-j} \cdot(-u)^{-j}
$$

и рассмотрим два уравнения

$$
\begin{aligned}
\chi_{+}\left(q^{-1} u\right) & =t_{+}(u) \chi_{+}(u)-G u^{2 L} \chi_{+}(q u), \\
\chi_{-}(q u) & =t_{-}(u) \chi_{-}(u)-G u^{-2 L} \chi_{-}\left(q^{-1} u\right),
\end{aligned}
$$

где $G \equiv q^{L} \lambda^{-2}$.

Пусть $\chi_{+}(u)$-голоморфное по $u$ решение уравнения (16) такое, что $\chi_{+}(0)=1$. Пусть $\chi_{-}(u)$ - голоморфное по $u^{-1}$ решение уравнения (17) такое, что $\chi_{-}(\infty)=1$. Обе эти функции определены однозначно и сушествуют для любых $t_{j}$ и $G$. Подставим, например, разложение

$$
\chi_{+}(u)=\sum_{n=0}^{\infty} \chi_{n} \cdot(-u)^{n}
$$

в (16) и выделим в этом выражении коэффициент при $u^{k}$. Условие равенства нулю этого коэффициента эквивалентно следуюшему условию: $\left(q^{-k}-1\right) \chi_{k}$ есть линейная комбинация величин $\chi_{n}$ при $0 \leqslant n<k$, коэффициентов $t_{+}$и $G$. Это, очевидно, простая рекурсия, однозначно фиксируемая начальным значением $\chi_{0}=1$. Более того, можно получить оценку сверху $\left|\chi_{n}\right|<|q|^{n^{2} / 4 L} X^{n}$, где несушественная величина $X$ определяется набором $t_{j}$ и $G$.

Кроме того, есть способ представить $\chi_{+}$как полубесконечное матричное произведение. Пусть

$$
L(u)=\left(\begin{array}{cc}
t_{+}(u) & -G u^{2 L} \\
1 & 0
\end{array}\right)
$$

$$
L_{n}(u)=L(u) L(q u)\left(q^{2} u\right) \ldots L\left(q^{n-1} u\right) .
$$

При $|q|<1$ матричное произведение $L_{n}(u)$ сходится абсолютно и

$$
L_{\infty}(u)=\left(\begin{array}{cc}
\chi_{+}\left(q^{-1} u\right) & 0 \\
\chi_{+}(u) & 0
\end{array}\right) .
$$

Это равенство следует из соотношения $L_{\infty}(u)=L(u) L_{\infty}(q u)$.

Аналогичные выражения могут быть написаны и для $\chi_{-}$. Например, если $t_{-}(u)=$ $t_{+}\left(u^{-1}\right)$ (т.е. $\left.T(u)=T\left(u^{-1}\right)\right)$, то $\chi_{-}(u) \equiv \chi_{+}\left(u^{-1}\right)$.

Замечательны квазиклассические пределы $\chi_{ \pm}(u)$. Функция $\psi(u)=\prod_{n=0}^{\infty}\left(1-q^{n} u\right)$ называется в некоторых приложениях "компактный квантовый дилогарифм", поскольку в пределе $q=e^{-\epsilon} \rightarrow 1$ она имеет главную асимптотику

$$
\psi(u)=\exp \left\{\epsilon^{-1} \int_{0}^{u} \ln (1-x) d \ln x+O(1)\right\} .
$$


В том же самом пределе голоморфное решение

$$
\chi\left(q^{-1} u\right)=t(u) \chi(u)-p(u) \chi(q u),
$$

где полиномы $t(u)$ и $p(u)$ таковы, что $t(0)=1$ и $p(0)=0$ (см. (16)), имеет асимптотику

$$
\chi(u)=\exp \left\{\epsilon^{-1} \int_{0}^{u} \ln y d \ln x+O(1)\right\},
$$

где $(x, y)$ - точка на гиперэллиптической кривой $y+p(x) y^{-1}=t(x)$. Начальная точка в интеграле $(23)$ есть $(x, y)=(0,1)$, а интегрирование ведется в окрестности этой точки.

\section{4. АНЗАЦ ДЛЯ ВОЛНОВОЙ ФУНКцИИ}

С учетом обозначений (11) функции

$$
\psi_{+}(x)=\frac{\chi_{+}(u)}{n_{+}(u)}, \quad \psi_{-}(x)=\frac{\chi_{-}(u)}{n_{-}(u)}
$$

являются решениями первого из уравнений (12), если

$$
\frac{n_{+}(u)}{n_{+}\left(q^{-1} u\right)}=-\lambda u^{-L}, \quad \frac{n_{-}(u)}{n_{-}(q u)}=-\lambda u^{L} .
$$

Решения второго из уравнений (12) суть

$$
\psi_{+}^{*}(x)=\frac{\chi_{+}^{*}(\tilde{u})}{n_{+}^{*}(\tilde{u})}, \quad \psi_{-}^{*}(x)=\frac{\chi_{-}^{*}(\tilde{u})}{n_{-}^{*}(\tilde{u})} .
$$

Уравнения (25) определяют класс функций $n_{+}$и класс функций $n_{-}$(т.е. $n_{ \pm}$определены с точностью до двоякопериодических функций), эти классы пока не фиксированы. Обшее решение уравнений (12) есть линейная комбинация четырех слагаемых:

$$
\Psi(x) \sim \sum_{\varepsilon, \varepsilon^{\prime}= \pm} \psi_{\varepsilon}(u) \psi_{\varepsilon^{\prime}}^{*}(\tilde{u}) .
$$

Можно оценить среднюю асимптотику каждого слагаемого. Пусть $f(x)$ при $x \rightarrow \pm \infty$ имеет асимптотические разностные свойства

$$
\frac{f(x)}{f(x+i b)} \sim a e^{2 \pi x b \varepsilon}, \quad \frac{f(x)}{f\left(x-i b^{-1}\right)} \sim a^{\prime} e^{2 \pi x b^{-1} \varepsilon^{\prime}},
$$

$\varepsilon$ и $\varepsilon^{\prime}$ - целые. С двиги в разностных уравнениях могут быть объединены в вешественный сдвиг $x \mapsto x+i\left(b-b^{-1}\right)$, а асимптотика $|f(x)|$ с вешественным $x \rightarrow \pm \infty$ может быть легко оценена как

$$
|f(x)| \leqslant F(x) \exp \left(\frac{\pi}{2}\left(\varepsilon+\varepsilon^{\prime}\right) \operatorname{cth} \theta x^{2}+\pi\left(\varepsilon+\varepsilon^{\prime}\right) \cos \theta x+\frac{\ln \left|a a^{\prime}\right|}{2 \sin \theta} x\right),
$$


где $F(x)$ - некоторая периодическая функция с периодом $i\left(b-b^{-1}\right)=-2 \sin \theta$. Усреднение асимптотики подразумевает замену $F(x) \mapsto \max F(x)=$ const. Отсюда заключаем, что $\psi_{ \pm}(u) \psi_{ \pm}^{*}(\tilde{u})$ растут при $x \rightarrow \pm \infty$, в то время как

$$
\left|\psi_{ \pm}(u) \psi_{\mp}^{*}(\tilde{u})\right| \sim e^{-2 \pi L \cos \theta|x|}, \quad x \rightarrow \pm \infty .
$$

Таким образом, мы приходим к следуюшему анзацу для $\Psi(x) \in L_{2}(\mathbb{R})$ :

$$
\Psi(x)=\frac{\chi_{+}(u) \chi_{-}^{*}(\tilde{u})}{D_{1}(x)}-\frac{\chi_{-}(u) \chi_{+}^{*}(\tilde{u})}{D_{2}(x)},
$$

где $D_{1} \sim n_{+} n_{-}^{*}$ и $D_{2} \sim n_{-} n_{+}^{*}$ удовлетворяют условиям

$$
\begin{array}{ll}
\frac{D_{1}(x)}{D_{1}(x-i b)}=-\lambda u^{-L}, & \frac{D_{1}(x)}{D_{1}\left(x-i b^{-1}\right)}=-\lambda^{*} \tilde{u}^{L}, \\
\frac{D_{2}(x)}{D_{2}(x+i b)}=-\lambda u^{L}, & \frac{D_{2}(x)}{D_{2}\left(x+i b^{-1}\right)}=-\lambda^{*} \tilde{u}^{-L} .
\end{array}
$$

Эти уравнения по-прежнему определяют классы функций. Предположим далее, что

$$
-\lambda=e^{i \pi L b^{2}-\pi \gamma b}, \quad G=e^{2 \pi \gamma b},
$$

где $\gamma \in \mathbb{R}$. Тогда $D_{1}$ и $D_{2}$ могут быть записаны в следующем виде:

$$
D_{1}(x)=e^{-i \pi L x^{2}+i \pi \gamma} u^{-L} W_{1}(u), \quad D_{2}(x)=e^{-i \pi L x^{2}-i \pi \gamma} u^{-L} W_{2}(u),
$$

где функции

$$
W_{1,2}(u)=u^{2 L} W_{1,2}(q u)
$$

суть $\left(x \mapsto x-i b^{-1}\right)$-периодические составные части $D_{1,2}$. Функции $W_{1}$ и $W_{2}$ определяются этими уравнениями по-прежнему с точностью до произвольных двоякопериодических функций. Согласно теореме Лиувилля уравнения (35) подразумевают, что $W_{1,2}(u)$ как функции $x$ имеют по крайней мере $2 L$ нулей в параллелограмме периодов. Конечно же, нули могут быть выбраны комплексными, так что $\Psi(x) \in L_{2}(\mathbb{R})$ и спектр $t_{j}$ произволен. Для квантования следует обратиться к условию аналитичности $\Psi(x)$ в полосе (14). Эта полоса включает в себя весь параллелограмм периодов, так что существует только один способ обеспечить аналитичность $\Psi(x)$ в полосе. Этот способ подразумевает следуюшее:

1) следует выбрать функцию $W_{2}$ пропорциональной $W_{1}$, при этом оба слагаемых в (31) имеют общий знаменатель и

$$
\Psi(x)=e^{i \pi L x^{2}} \frac{e^{-i \pi \gamma x} \chi_{+}(u) \chi_{-}^{*}(\tilde{u})-\xi e^{i \pi \gamma x} \chi_{-}(u) \chi_{+}^{*}(\tilde{u})}{u^{-L} W(u)}
$$

где $\xi$ - некоторое комплексное число, $W_{1}=W_{2}=W$; 
2) функция $W(u)$ должна быть выбрана с минимально возможным набором из $2 L$ нулей:

$$
W(u)=\prod_{j=1}^{2 L} H\left(u / s_{j}\right),
$$

где $H(u)=-u H(q u)-$ тета-функция,

$$
H(u)=(u ; q)_{\infty}\left(q u^{-1} ; q\right)_{\infty}(q ; q)_{\infty}=\sum_{n \in \mathbb{Z}} q^{n(n-1) / 2}(-u)^{n},
$$

так что уравнения (35) приводят к ограничению

$$
\prod_{j=1}^{2 L} s_{j}=1
$$

3) необходимо обеспечить сокрашение нулей знаменателя с нулями числителя в формуле (36) во всей полосе. Этот прием использовался Гутсвиллером [3].

Последнее условие порождает бесконечную систему уравнений для набора из $2 L-1$ независимых переменных $s_{j}, j=1, \ldots, 2 L-1$ и $2 L-1$ параметров $t_{j}, j=1, \ldots, 2 L-1$. K счастью, сушествует специальный выбор $s_{j}$, который позволяет свести бесконечную систему уравнений к конечной системе, и это будет сделано в следуюшем разделе.

\section{5. УРАВНЕНИЯ КВАНТОВАНИЯ}

Помимо обозначений (11) и (33) удобно ввести также величины

$$
s_{j}=e^{2 \pi \sigma_{j} b}, \quad \sum_{j=1}^{2 L} \sigma_{j}=0 .
$$

Нули знаменателя в равенстве (36) в полосе (14) суть точки $\sigma_{j}+i n b-i n b^{-1}, j=$ $1, \ldots, 2 L, n \in \mathbb{Z}$. Им соответствуют $u=q^{n} s_{j}$ и $\tilde{u}=q^{* n} \tilde{s}_{j}$. Условие равенства нулю числителя (36) в этих точках дает бесконечный набор уравнений

$$
\xi=e^{-2 \pi i \gamma \sigma_{j}} \frac{G^{n} \frac{\chi+\left(q^{n} s_{j}\right)}{\chi-\left(q^{n} s_{j}\right)}}{G^{* n} \frac{\chi_{+}^{*}\left(q^{* n} \tilde{s}_{j}\right)}{\chi_{-}^{*}\left(q^{* n} \tilde{s}_{j}\right)}}, \quad n \in \mathbb{Z}, \quad j=1, \ldots, 2 L .
$$

Числитель и знаменатель в каждом из этих уравнений сопряжены, и более тщательный анализ показьвает, что все эти уравнения можно удовлетворить, если и числитель, и знаменатель не зависят от $n$, т.е.

$$
G^{n} \frac{\chi_{+}\left(q^{n} s_{j}\right)}{\chi_{-}\left(q^{n} s_{j}\right)}=\frac{\chi_{+}\left(s_{j}\right)}{\chi_{-}\left(s_{j}\right)} \stackrel{\text { def }}{=} M\left(s_{j}\right) .
$$

Независимость от $n$ может быть переписана следуюшим образом. Пусть

$$
w(u) \stackrel{\text { def }}{=} \chi_{+}\left(q^{-1} u\right) \chi_{-}(u)-G \chi_{+}(u) \chi_{-}\left(q^{-1} u\right) .
$$


Тогда условие (42) может быть переписано как $w\left(q^{n} s_{j}\right)=0$ для любых $n, j$.

Функция $w$ связана с $q$-вронскианом $\psi_{+}\left(q^{-1} u\right) \psi_{-}(u)-\psi_{+}(u) \psi_{-}\left(q^{-1} u\right)$ уравнения (24). Исключая отсюда $n_{ \pm}$, можно получить равенство (43). Из уравнений $(16),(17)$ для $\chi_{ \pm}$можно получить, что $w(u)=u^{2 L} w(q u)$, и поскольку (43) по определению есть сходящийся ряд по $u, u^{-1}$, то сушествует единственный набор $s_{j}, j=1, \ldots, 2 L$, такой, что

$$
w(u)=C \prod_{j=1}^{2 L} H\left(u / s_{j}\right), \quad \prod_{j=1}^{2 L} s_{j}=1 .
$$

Константа $C$, зависяшая от $G$ и набора $t_{j}$, несушественна, и $W(u)=w(u) / C$.

Следовательно, если $s_{j}$ являются нулями вронскиана (43), то весь бесконечный набор уравнений (41) приводится к конечному набору из $2 L-1$ уравнений (поскольку число $\xi$ само по себе должно определяться этими уравнениями)

$$
\xi=e^{-2 \pi i \gamma \sigma_{j}} \frac{M\left(s_{j}\right)}{M^{*}\left(\tilde{s}_{j}\right)}, \quad j=1, \ldots, 2 L .
$$

Это система уравнений на коэффициенты $t_{j}$ "потенциала" (8), число уравнений в точности совпадает с числом неизвестных $t_{j}$ и с числом независимых $s_{j}$. Из формул (43), (44) следует, что $s_{j}$ есть однозначная функция от набора $t_{j}$. С другой стороны, можно показать, что $t_{j}$ суть многозначные функции от $s_{j}$ с бесконечным числом листов, что и обеспечивает бесконечное число решений уравнений (45).

Возвращаясь к концу предыдущего раздела, можно убедиться, что условие (37) выполняется автоматически, так что оно не является гипотезой, а следует из (41) и последующего анализа.

Численные исследования уравнений (45) позволяют предположить, что все их решения соответствуют вешественным $\sigma_{j}$, так что $\xi$ - чистая фаза. При этом $\Psi(x)$ из $(36)$ имеет постоянную фазу при $x \in \mathbb{R}$ (поскольку комплексное сопряжение эквивалентно модулярному преобразованию). Кроме того, если "потенциал" симметричен, $T(u)=$ $T\left(u^{-1}\right)$, то $\xi= \pm 1-$ просто четность.

Заметим в заключение, что благодаря определению (42), числитель в (36) равен нулю в точках $\sigma_{j}+i n b-i m b^{-1}, n, m \in \mathbb{Z}$, так что $\Psi(x)$ аналитична во всей комплексной плоскости.

\section{6. КВАНТОВАЯ РЕЛЯТИВИСТСКАЯ ЦЕПОЧКА ТОДЫ}

Квантовая кривая для квантовой релятивистской цепочки Тоды длины $N$ в одной из возможных нормировок [4] есть

$$
\mathbf{J}(\mathbf{u}, \mathbf{v})=\mathbf{v}+(-)^{N} G \mathbf{u}^{N} \mathbf{v}^{-1}-t(\mathbf{u}),
$$

где

$$
t(u)=\sum_{j=0}^{N} t_{j}(-u)^{j}, \quad t_{0}=t_{N}=1 .
$$


Уравнения Бакстера для $Q(x)=\langle Q \mid x\rangle$ суть $\langle Q| \mathbf{J}=\langle Q| \mathbf{J}^{\dagger}=0$.

Условия для $Q(x)$ следуюшие [2], [5]:

1) $|Q(x)| \sim 1$ при $x \rightarrow-\infty$ и $|Q(x)| \sim e^{-2 \pi N \cos \theta x}$ при $x \rightarrow+\infty$;

2) $Q(x)$ - целая функция.

Слегка модифицируя предыдущие рассуждения (они соответствуют случаю четных $N=2 L$ ), определим $\chi_{ \pm}$как голоморфные по $u^{ \pm 1}$ решения:

$$
\begin{aligned}
\chi_{+}\left(q^{-1} u\right) & =t(u) \chi_{+}(u)-G(-u)^{N} \chi_{+}(q u), \\
\chi_{-}(q u) & =\frac{t(u)}{(-u)^{N}} \chi_{-}(u)-\frac{G}{(-u)^{N}} \chi_{-}\left(q^{-1} u\right) .
\end{aligned}
$$

Их $q$-вронскиан определяется формулой (43), при этом имеют место разностное свойство $W(u)=(-u)^{N} W(q u)$ и разложение

$$
W(u)=C \prod_{j=1}^{N} H\left(u / s_{j}\right), \quad \prod_{j=1}^{N} s_{j}=1 .
$$

Пусть, как и в предыдуших разделах,

$$
M\left(s_{j}\right)=\frac{\chi_{+}\left(s_{j}\right)}{\chi_{-}\left(s_{j}\right)},
$$

и условие квантования для вешественных $\sigma_{j}, s_{j} \equiv e^{2 \pi \sigma_{j} b}$ есть

$$
e^{-2 \pi i \gamma \sigma_{j}} \frac{M\left(s_{j}\right)}{M\left(s_{j}\right)^{*}}=\xi, \quad j=1, \ldots, N .
$$

Все обозначения здесь те же, что и использованные ранее.

Теперь функция $Q(x)$, удовлетворяюшая всем условиям, задается формулой

$$
Q(x)=\frac{e^{-2 \pi i \gamma x} \chi_{+}(u) \chi_{-}^{*}(\tilde{u})-\xi \chi_{-}(u) \chi_{+}^{*}(\tilde{u})}{W(u)} .
$$

\section{7. ЗАКЛЮЧЕНИЕ}

В этой работе мы предлагаем сценарий квантования для некоторых уравнений типа уравнения Бакстера в режиме сильной связи. Первой важной деталью сценария является то, что определенные $q$-разностные уравнения второго порядка имеют голоморфные по $u$ решения (функции $\chi$ ) подобно тому, как компактньй квантовый дилогарифм является голоморфным решением разностного уравнения первого порядка [6]. Эти функции однозначно определены и замечательно подходят для численного анализа, это отличает наш случай от случая $|q|=1$ [5]. Другое важное наблюдение заключается в том, что $q$-определитель Вронского двух функций $\chi$ есть произведение тета-функций, и именно вронскиан должен быть использован для преобразования функций $\chi$ в решения исходных разностных уравнений. Использование нулей вронскиана сводит бесконечную систему уравнений на вычеты к конечной. 
Условие квантования, т.е. условие аналитичности волновой функции или функции Бакстера $Q$ в полосе (14), приводится к набору трансцендентных уравнений (45) в терминах функций $\chi$. Число уравнений в (45) растет при росте размера системы. Мы до сих пор не нашли хорошего метода для исследования этих уравнений в термодинамическом пределе.

В этой работе мы рассматривали спешифические квантовые кривые $\mathbf{J}(\mathbf{u}, \mathbf{v})$ гиперэллиптического типа (7) и (46). В обоих примерах $\Psi(x)$ и $Q(x)$ оказались аналитическими функциями во всей комплексной плоскости, это особенность $\mathbf{J}(\mathbf{u}, \mathbf{v})$ тодовского типа. В более сложных примерах это не обязательно так. Предложенная схема может быть обобщена на весь класс квантовых алгебраических кривых (не только гиперэллиптических), соответствующий классу интегрируемых моделей с локальной алгеброй Вейля как алгеброй наблюдаемых [7].

Благодарности. Эта работа поддержана Исследовательским Советом Австралии (ARC) и частично грантом CGP CRDF RM1-2334-MO-02. Я хотел бы поблагодарить В. Бажанова, Р. Кашаева, С. Пакуляка и М. Семенова-Тян-Шанского за многочисленные полезные обсуждения.

\section{Список литературы}

[1] L. D. Faddeev. Lett. Math. Phys. 1995. V. 34. P. 249-254; L. D. Faddeev. Modular double of quantum group. In: Conférence Moshé Flato 1999. Quantization, Deformation and Symmetries. Dijon, France, September 5-8, 1999. V. 1. Math. Phys. Stud. V. 21. Eds. G. Dito, D. Sternheimer. Dordrecht: Kluwer, 2000. P. 149-156; L. D. Faddeev, R. M. Kashaev, A. Yu. Volkov. Commun. Math. Phys. 2001. V. 219. P. 199-219; L. D. Faddeev, R. M. Kashaev. J. Phys. A. 2002. V. 35. P. 4043-4048.

[2] S. Kharchev, D. Lebedev, M. Semenov-Tian-Shansky. Commun. Math. Phys. 2002. V. 225. P. 573-609.

[3] M. Gutzwiller. Ann. of Phys. 1981. V. 133. P. 304-331; V. Pasquier, M. Gaudin. J. Phys. A. 1992. V. 25. P. 5243-5252; S. Kharchev, D. Lebedev. Lett. Math. Phys. 1999. V. 50. P. 53-77.

[4] V. Kuznetsov, A. Tsiganov. Separation of variables for the quantum relativistic Toda lattices. hep-th/9402111; G. Pronko, S. Sergeev. J. Appl. Math. 2001. V. 1. P. 47-68.

[5] F. A. Smirnov. Dual Baxter equations and quantization of Affine Jacobian. math-ph/0001032; Baxter equations and Deformation of Abelian Differentials. math-ph/0302014.

[6] L. D. Faddeev, R. M. Kashaev. Mod. Phys. Lett. A. 1994. V. 9. P. 427-434.

[7] S. M. Sergeev. J. Phys. A. 1999. V. 32. P. 5693-5714; C. М. Сергеев. ТМФ. 2000. Т. 124. № 3. C. 391-409. 